\title{
The comparison of predictive performance in bispectral index prediction during target effect-site controlled infusion of propofol using different blood effect-site equilibration rate constants in the same pharmacokinetic model
}

\author{
Byung-Moon Choi ${ }^{1}$, Ji-Youn Bang ${ }^{1}$, Kyeo-Woon Jung ${ }^{1}$, Ju-Hyun Lee ${ }^{1}$, Heon-Yong Bae ${ }^{1}$, and \\ Gyu-Jeong Noh ${ }^{1,2}$
}

Departments of ${ }^{1}$ Anesthesioloy and Pain Medicine, ${ }^{2}$ Clinical Pharmacology and Therapeutics, University of Ulsan College of Medicine, Asan Medical Center, Seoul, Korea

Background: Blood-brain equilibration rate constant $\left(k_{e 0}\right)$ is derived from either pharmacokinetic and pharmacodynamic modeling $\left(k_{\text {eo_model }}\right)$ or a model-independent observed time to peak effect $\left(k_{\text {e0_tpeak }}\right)$. Performance in bispectral index (BIS) prediction was compared between $k_{e 0_{-} \text {model }}$ and $k_{e 0_{-} \text {tpeak }}$ for microemulsion or long chain triglyceride (LCT) propofol.

Methods: Time to peak effect ( $\mathrm{t}_{\text {peak }}$, time to a maximally reduced BIS value) of microemulsion propofol after an intravenous bolus $\left(1 \mathrm{mg} / \mathrm{kg}\right.$ ) was measured in 100 patients (group $\mathrm{A}_{\text {micro }}$ ). An observed $\mathrm{t}_{\text {peak }}$ of $1.6 \mathrm{~min}$ for LCT propofol was obtained from an earlier study. Another 40 patients received a target controlled infusions of microemulsion propofol $\left(k_{e 0 \_ \text {model }}\right.$ $=0.187 / \mathrm{min}$, group $\left.\mathrm{B}_{\text {micro }}=20\right)$ or LCT propofol $\left(k_{e 0 \_ \text {model }}=0.26 / \mathrm{min}\right.$, group $\left.\mathrm{B}_{\mathrm{LCT}}=20\right)$ and remifentanil. The $k_{e 0 \_ \text {tpeak }}$ s in group $\mathrm{B}_{\text {micro }}$ and $\mathrm{B}_{\mathrm{LCT}}$ were calculated using the observed $t_{\text {peak }}$ value obtained from group $\mathrm{A}_{\text {micro }}$ and $1.6 \mathrm{~min}$, respectively. Effectsite concentrations of propofol were recalculated using the amounts of propofol infused over time and $k_{e 0 \_ \text {tpeak }}$ 's. Predicted BIS values calculated by sigmoid Emax equations with $k_{e 0 \_ \text {model }}$ and $k_{e 0_{\text {_tpeak }}}$ were compared with observed BIS values during induction and emergence for both formulations of propofol.

Results: Observed $\mathrm{t}_{\text {peak }}$ of microemulsion propofol was $1.68 \mathrm{~min}$. The median performance errors of BIS in group $\mathrm{B}_{\text {micro }}$ were $-1.83 \%$ ( -24.8 to $\left.18.9, k_{e 0 \_ \text {model }}\right)$ and $-2.42 \%$ ( -26.1 to $\left.36.2, k_{e 0_{\text {tpeak }}}\right)$, while $8.01 \%\left(-20.5\right.$ to $\left.30.1, k_{e 0_{-} \text {model }}\right)$ and $7.37 \%$ $\left(-27.0\right.$ to $\left.49.1, k_{e 0_{-} \text {tpeak }}\right)$ in group $\mathrm{B}_{\mathrm{LCT}}$. The median absolute performance errors of BIS in group $\mathrm{B}_{\text {micro }}$ were $11.87 \%(2.2-$ $\left.31.1 k_{e 0_{-} \text {model }}\right)$ and $14.38 \%\left(-0.6\right.$ to $\left.44.6, k_{e 0_{-} \text {tpeak }}\right)$, while $17.31 \%\left(5.54-36.0, k_{e 0_{-} \text {model }}\right)$ and $18.28 \%\left(-0.1\right.$ to $\left.56.0, k_{e 0_{-} \text {tpeak }}\right)$ in group $\mathrm{B}_{\mathrm{LCT}}$.

Conclusions: The $k_{e 0 \_ \text {model }}$ showed better performance in BIS prediction than the $k_{e 0_{\text {tpeak }} \text {. }}$ (Korean J Anesthesiol 2013; 65: 299-305)

Key Words: Bispectral index, Pharmacokinetic, Propofol.

\footnotetext{
Received: December 12, 2012. Revised: 1st, March 10, 2013; 2nd, April 11, 2013. Accepted: April 24, 2013.

Corresponding author: Gyu-Jeong Noh, M.D., Ph.D., Department of Anesthesioloy and Pain Medicine, Asan Medical Center, 388-1, Pungnap 2-dong, Songpa-gu, Seoul 138-736, Korea. Tel: 82-2-3010-3855, Fax: 82-2-3010-6790, E-mail: nohgj@amc.seoul.kr

(c) This is an open-access article distributed under the terms of the Creative Commons Attribution Non-Commercial License (http:// creativecommons.org/licenses/by-nc/3.0/), which permits unrestricted non-commercial use, distribution, and reproduction in any medium, provided the original work is properly cited.
} 


\section{Introduction}

Target-controlled infusion (TCI) systems were developed to administer intravenous agents during anesthesia. This is a logical approach for the fine titration of intravenous hypnotics and opioids and is based on the pharmacokinetic model that describes the disposition of the drug. For targeting effect-site concentrations, the TCI system should incorporate pharmacokinetic parameters, such as the distribution volume and clearance, and the blood-brain equilibration rate constant $\left(k_{e 0}\right)$. Pharmacokinetic parameters can be determined by assessing the time course of the drug. On the other hand, $k_{e 0}$ can be estimated using pharmacokinetic/pharmacodynamic modeling of a single population $\left(k_{\text {e0_model }}\right)$ or by calculating model-independent time-to-peak effects $\left(k_{e 0 \_ \text {tpeak }}\right)[1,2]$. Although integrated pharmacokinetic/pharmacodynamic modeling of a single population is the standard method for obtaining $k_{e 0}$, this is complicated by the need for blood samples and the frequent measurement of drug effects. As a result, the pharmacokinetic model for propofol that was reported by Marsh et al. [3], a popular model used in clinical situations, only estimates pharmacokinetic parameters without $k_{e 0}$.

The time of peak effect after bolus injection, $t_{\text {peak }}$, could be used to link separate pharmacokinetic and pharmacodynamic studies [2]. The $t_{\text {peak }}$ method is based on the assumption that the time required to reach the maximal effect-site concentration is identical to the time required to produce the maximal drug effects. Minto et al. [2] reported that the $t_{\text {peak }}$ method, which is an alternative approach that can be used to obtain $k_{e 0}$, can also be used to obtain better approximations of the postulated "true" time course of propofol effects in comparison with the naive method. The observed $t_{\text {peak }}$ of propofol was 1.6 minutes in a previous study [1], which was then used to calculate $k_{e 0}$ using differential equations. The $k_{e 0}$ of long-chain triglyceride (LCT) propofol, which was calculated as $1.2152 / \mathrm{min}$ using the $t_{\text {peak }}$ method, is included the Orchestra workstation (Fresenius Kabi AG, Germany) and was previously modified by Marsh et al. [4].

Microemulsion propofol (Aquafol; Daewon Pharmaceutical Co., Ltd, Seoul, Korea) was originally developed to overcome the drawbacks of LCT propofol [5]. Sequential pharmacokinetic/pharmacodynamic modeling of microemulsion propofol was performed in healthy volunteers [6]. The comparison of appropriateness between $k_{e 0}$ s derived from different methods in target effect-site concentration controlled infusion of propofol has yet to be fully elucidated. The aim of this study is to compare predictive performance in bispectral index (BIS, Aspect Medical Systems, Newton, MA, USA) prediction during target effect-site controlled infusion of propofol using different $k_{e 0}$ in the same pharmacokinetic model.

\section{Materials and Methods}

\section{Part 1: Patient population and study procedure used to determine the $k_{e 0}$ value of microemulsion propofol using the $t_{\text {peak }}$ method}

After obtaining approval from the Institutional Review Board of our institution and signed informed consent from all patients, a total of 100 patients diagnosed as American Society of Anesthesiologist (ASA) physical status (PS) 1 or 2 who were scheduled for stomach or colorectal surgery under general anesthesia were enrolled in this study. Patients with known or suspected cardiac, liver, or renal disease, significant obesity ( $>120 \%$ of ideal weight), patients currently receiving sedative or analgesic drugs, and patients with known adverse effects to propofol were excluded. The patient characteristics are shown in Table 1. All patients were required to fast starting the midnight before the operation without receiving premedication. Once in the operating room, patients were monitored using electrocardiography, pulse oximetry, end-tidal carbon dioxide partial pressure, noninvasive blood pressure monitoring (Datex-Ohmeda S/5; Planar Systems, Inc., Beaverton, USA), and BIS. Using RS232C cables, all data were continuously downloaded onto a personal computer until recovery from anaesthesia. An 18-gauge catheter was inserted into a large vein in the wrist. Forty milligrams of lidocaine were intravenously administered prior to propofol injection, then an intravenous bolus of $1 \mathrm{mg} / \mathrm{kg}$ microemulsion propofol was administered. After observing the maximal reduction in BIS from baseline, propofol and remifentanil were administered by effect-site target-controlled infusion (Asan Pump, version 2.1.3; Bionet Co., Ltd., Seoul, Korea).

Table 1. Patient Characteristics

\begin{tabular}{lccc}
\hline & & \multicolumn{2}{c}{ Part 2 } \\
\cline { 3 - 4 } & $\begin{array}{c}\text { Part } 1 \\
(\mathrm{n}=100)\end{array}$ & $\begin{array}{c}\text { Microemulsion } \\
\text { propofol }(\mathrm{n}=20)\end{array}$ & $\begin{array}{c}\text { LCT propofol } \\
(\mathrm{n}=20)\end{array}$ \\
\hline Age $(\mathrm{yr})$ & $52.0(46.0-62.0)$ & $59.2 \pm 10.2$ & $60.2 \pm 10.2$ \\
Height $(\mathrm{cm})$ & $161.5 \pm 8.1$ & $159.7 \pm 9.6$ & $163.4 \pm 5.0$ \\
Weight $(\mathrm{kg})$ & $59.7(54.6-67.6)$ & $59.2 \pm 10.7$ & $63.8 \pm 13.8$ \\
Sex (M/F) & $52 / 48$ & $7 / 13$ & $15 / 5$ \\
ASA PS (I/II) & $22 / 78$ & $3 / 17$ & $0 / 20$ \\
\hline
\end{tabular}

Parts 1 and 2 of this study were designed to determine the $k_{e 0}$ value for microemulsion propofol using the $t_{\text {peak }}$ method. The predictive performance of BIS was determined during TCI using the $2 k_{e 0} \mathrm{~s}$ values used in the same pharmacokinetic propofol model. Data are expressed as the mean \pm standard deviation, median (range: $25-75 \%$ ), or countswhere appropriate. LCT: long-chain triglyceride, ASA PS: American Society of Anesthesiologists physical status. 
The differential equation describing effect-site concentration as a function of time $t$ was presented as equation (1)

$$
\frac{d C e(t)}{d t}=k_{e 0} \cdot C p(t)-k_{e 0} \cdot C e(t)=k_{e 0}(C p(t)-C e(t))
$$

Equation 2 shows the Laplace transform of Equation (1).

$$
\begin{aligned}
& s C e(s)=k_{e 0} \cdot C p(s)-k_{e 0} \cdot C e(s) \\
& C e(s)=\frac{k_{e 0}}{s+k_{e 0}} C p(s)
\end{aligned}
$$

Equation 3 shows the inverse Laplace transform of the conversion of the time domain.

$$
C e(t)=k_{e 0} \cdot e^{-k_{e 0} \cdot t} * C p(t)
$$

Equation 4 shows the unit disposition function of the plasma concentration determined in a 3-compartment mammillary model.

$$
C p(t)=\sum_{i=1}^{3} C_{i} e^{-\lambda_{i} t}
$$

Starting with Equation 3, the convolution of the input and disposition functions is shown in Equation 5.

$$
\begin{aligned}
& C e(t)=k_{e 0} \int_{0}^{t} e^{-k_{e 0} \cdot(t-\tau)} C p(\tau) d \tau \\
& =k_{e 0} \int_{0}^{t} e^{-k_{e 0}(t-\tau)} C_{i} e^{-\lambda_{i} \cdot \tau} d \tau \\
& =C_{i} \cdot k_{e 0} \cdot e^{-k_{e 0} \cdot t} \int_{0}^{t} e^{k_{e 0} \cdot \tau} e^{-\lambda_{i} \cdot \tau} d \tau=C_{i} \cdot k_{e 0} \cdot e^{-k_{e 0} \cdot t} \int_{0}^{t} e^{\left(k_{e 0}-\lambda_{i}\right) \cdot \tau} d \tau \\
& =C_{i} \cdot k_{e 0} \cdot e^{-k_{e 0} \cdot t}\left[\frac{e^{\left(k_{e 0}-\lambda_{i}\right) \cdot \tau}}{k_{e 0}-\lambda_{i}}\right]_{0}^{t}=C_{i} \cdot k_{e 0} \cdot e^{-k_{e 0} \cdot t} \cdot\left(\frac{e^{\left(k_{e 0}-\lambda_{i}\right) \cdot t}-1}{k_{e 0}-\lambda_{i}}\right) \\
& =C_{i} \cdot k_{e 0} \cdot \frac{e^{-k_{e 0} \cdot t} \cdot e^{\left(k_{e 0}-\lambda_{i}\right) \cdot t}-e^{-k_{e 0} \cdot t}}{k_{e 0}-\lambda_{i}}=C_{i} \cdot k_{e 0} \cdot \frac{e^{-\lambda_{i} \cdot t}-e^{-k_{e 0} \cdot t}}{k_{e 0}-\lambda_{i}}
\end{aligned}
$$

As a result of Equation 5, the effect-site concentration of the 3-compartment mammillary model can be summarized using Equation 6.

$$
C e(t)=\sum_{i=1}^{3} \frac{k_{e 0} \cdot C_{i}}{k_{e 0}-\lambda_{i}}\left(e^{-\lambda_{i} \cdot t}-e^{-k_{e 0} \cdot t}\right) \quad \text { (Eq.6), where Ci and } \lambda_{\mathrm{i}}
$$

Table 2. Pharmacokinetic Parameters and $k_{e 0}$ s Values of the Noh and Modified Marsh Models

\begin{tabular}{lcc}
\hline & Noh model & Modified Marsh model \\
\hline$V_{1}(\mathrm{~L})$ & 21.0 & $0.228 \times \mathrm{WT}$ \\
$V_{2}(\mathrm{~L})$ & $86.7+(\mathrm{LBM} / 47)^{11.3}$ & $0.473 \times \mathrm{WT}$ \\
$V_{3}(\mathrm{~L})$ & 757 & $2.865 \times \mathrm{WT}$ \\
$C L(\mathrm{~L} / \mathrm{min})$ & $1.37+0.0091 \times(\mathrm{WT}-64)$ & $0.027 \times \mathrm{WT}$ \\
$Q_{1}(\mathrm{~L} / \mathrm{min})$ & $\mathrm{F}: 0.855 ; \mathrm{M}: 1.17$ & $0.026 \times \mathrm{WT}$ \\
$Q_{2}(\mathrm{~L} / \mathrm{min})$ & 0.807 & $0.010 \times \mathrm{WT}$ \\
$k_{e 0}(/ \mathrm{min})$ & $0.187^{*}$ & $1.2152^{\dagger}$ \\
\hline
\end{tabular}

F: female, M: male, LBM: lean body mass calculated using the James formula, WT: weight. *Estimated using sequential pharmacokinetic/ pharmacodynamic modeling of a single population. ${ }^{\dagger}$ Calculated based on the observed time-to-peak effects. are the coefficients and exponents of the pharmacokinetic model, respectively.

Because $t_{\text {peak }}$ is the time at which effect-site concentration (Ce) reaches its maximal value, the derivative of $\mathrm{Ce}$ with respective to $\mathrm{t}$ should be 0 at $\mathrm{t}=\mathrm{t}_{\text {peak }}$.

$$
C e(t)=\sum_{i=1}^{3} \frac{k_{e 0} \cdot C_{i}}{k_{e 0}-\lambda_{i}}\left(\lambda_{i} e^{-\lambda_{i} \cdot t_{p e a k}}-k_{e 0} e^{-k_{e 0} \cdot t_{\text {peak }}}\right)=0
$$

Thus, Equation 6 can be used to mathematically determine $k_{e}$.

Part 2: Patient population and study procedure used to compare BIS predictions during $\mathrm{TCl}$ based on the $2 k_{e o}$ values determined in pharmacokinetic propofol models

A total of 40 ASA PS $1 / 2$ patients who were scheduled for stomach, breast, or colorectal surgery were randomly divided and assigned to receive microemulsion $(n=20)$ or LCT propofol ( $n=20$; Pofol, Jeil Pharmaceutical Co., Ltd, Seoul, Korea). The patient characteristics are shown in Table 1. All patients were required to fast starting the midnight before surgery without receiving any premedication. Once in the operating room, patients were monitored using electrocardiography, pulse oximetry, end-tidal carbon dioxide partial pressure, noninvasive blood pressure monitoring, and BIS. Using RS232C cables, these data were continuously downloaded onto a personal computer until recovery from anaesthesia. Each patient was preoxygenated with $100 \%$ oxygen via a facemask. Microemulsion propofol was administered via effect-site target-controlled infusion based on the Noh model, and a modified version of the Marsh model was used to administer LCT propofol to the other group of patients. The pharmacokinetic parameters and $k_{e 0}$ values of the Noh and modified Marsh models are shown in Table 2 [4,6]. Remifentanil were administered via effect-site target-controlled infusion based on the Minto model [7]. Propofol and remifentanil were infused using TCI software, and the infusion histories of the patients were automatically recorded onto comma separated values (.csv) files. The pharmacokinetic parameters and $k_{e 0}$ values shown in Table 2 were programmed into the Asan pump. Tracheal intubation was facilitated by administering $0.12 \mathrm{mg} /$ $\mathrm{kg}$ vecuronium. Patients were then ventilated with a mixture of oxygen and air (delivered at a $1: 2$ ratio), and the ventilation rate was adjusted to maintain the end-tidal carbon dioxide partial pressure between 35-45 mmHg. The target site-effective concentration of propofol was adjusted to maintain BIS values $<60$. Target site-effective concentrations of remifentanil were titrated to maintain stable hemodynamics (i.e., systolic blood pressure $[\mathrm{SBP}]>80 \mathrm{mmHg}$ and heart rate $[\mathrm{HR}]>45$ beats/min). If necessary, ephedrine or atropine was administered to maintain SBP $>80 \mathrm{mmHg}$ and HR > 45 beats/min during anesthesia. Neuro- 
muscular blockade was reversed by administering neostigmine and glycopyrrolate at the end of surgery.

\section{Part 3: Simulation based on the infusion propofol history described in Part 2}

The infusion history of propofol determined in Part 2 was used to simulate the effect-site concentration of propofol using different $k_{e 0}$ values in same pharmacokinetic model. In part 2 , patients received microemulsion propofol using TCI with $k_{e 0}$ obtained by $k_{e 0 \_ \text {estimation }}$ method. On the other hand, patients received LCT propofol using TCI with $k_{e 0}$ obtained by $k_{e 0_{-} \text {tpeak }}$ method. For simulation, $k_{e 0}$ s of microemulsion and LCT propofol was changed to different $k_{e 0}$ s obtained by $k_{e 0 \_ \text {tpeak }}$ method for microemulsion propofol and $k_{e 0 \_ \text {estimation }}$ method for LCT propofol. $k_{e 0 \_ \text {estiation }}$ value for LCT propofol was obtained from the result of Billard et al. [8]. After each csv files was loaded in Asan Pump, $k_{e 0}$ s of propofol were changed to $0.26 / \mathrm{min}$ for LCT propofol and result of part 1 for microemulsion propofol [8], and simulations of individual patients were performed by Asan Pump. Model summary for TCI and simulation of microemulsion and LCT propofol is shown in Table 3.

\section{Predictive performance}

In accordance with a previous method [9], the predictive performance of the TCI system was determined using the pharmacokinetic parameters and the $k_{e 0}$ values for each type of propofol were characterized based on 2 parameters: bias and inaccuracy. BIS values (measured from the induction of propofol infusion to intubation) and recovery periods (measured from the discontinuation of propofol infusion to extubation) were used to analyze the results of the present study. For each BIS value, the performance error (PE) of the ith patient was calculated as follows:

$$
\mathrm{PE}_{\mathrm{ij}}=\frac{\text { measured }_{\mathrm{ij}}-\operatorname{predicted}_{\mathrm{ij}}}{\operatorname{predicted}_{\mathrm{ij}}}
$$

where predicted $_{\mathrm{ij}}$ represents the predicted BIS value at the $j$ th sampling point of the $i$ th patient and measured $_{\mathrm{ij}}$ is the observed BIS value.
The sampling interval $\left(t_{j}-t_{j-1}\right)$ for BIS measurement was 10 seconds. The predicted value of BIS for each sample was calculated using the inhibitory sigmoid $\mathrm{E}_{\max }$ model:

$$
\text { Effect }=E_{0}+\left(E_{\max }-E_{0}\right) \frac{C e^{\gamma}}{C e_{50}^{\gamma}+C e^{\gamma}}
$$

where Effect is the BIS value, $E_{0}$ is the baseline BIS value when no drug is present, $E_{\max }$ is the maximal possible drug effect on $\mathrm{BIS}, \mathrm{Ce}$ is the predicted site-effective concentration of propofol determined in Part 3, $\mathrm{Ce}_{50}$ is the site-effective concentration associated with $50 \%$ of the maximal drug effect on BIS, and $\gamma$ is the steepness of the site-effective concentration vs the BIS relationship.

Various pharmacodynamic parameters for microemulsion and LCT propofol, such as $E_{0}, E_{\max }, C e_{50}$, and $\gamma$, were determined in our previous studies [5,6]. The first performance measure, the bias (i.e., the direction and size of deviation from the target) of the ith individual, was calculated as the median performance error (MDPEi) as follows:

$$
M D P E_{i}=\operatorname{median}\left\{P E_{i j}, j=1, \ldots \ldots . N_{i}\right\}
$$

The inaccuracy of BIS prediction (i.e., the size of the typical miss) of the $i$ th individual was calculated as the median absolute performance error $\left(\mathrm{MDAPE}_{\mathrm{i}}\right)$ as follows:

$$
M D A P E_{i}=\operatorname{median}\left\{\left|P E_{i j}\right|, j=1, \ldots \ldots . N_{i}\right\}
$$

where $N_{i}$ is the number of blood sampling points for the $i$ th individual.

The pooled-data approach was used to obtain population estimates for bias and inaccuracy (fit4NM 3.5.1, Eun-Kyung Lee and Gyu-Jeong Noh, http://www.fit4nm.org/download, last accessed: Oct 17, 2011).

\section{Statistical analysis}

All statistical analyses were conducted using R software (version 2.13.1; R Foundation for Statistical Computing, Vienna, Austria) or SigmaStat 3.5 for Windows (Systat Software, Inc., Chicago, IL). Data are expressed as the mean \pm standard deviation (SD) for normally distributed continuous variables, the

Table 3. Model Summary of the Target-controlled Infusion and Simulated Infusion of Microemulsion Propofol and Long-chain Triglyceride Propofol

\begin{tabular}{lcc}
\hline & Microemulsion propofol $(\mathrm{n}=20)$ & LCT propofol $(\mathrm{n}=20)$ \\
\hline Pharmacokinetic model of propofol & Noh model & Modified Marsh model \\
Pharmacokinetic model of remifentanil & Minto model & Minto model \\
$k_{e 0}$ for target-controlled infusion $(\mathrm{min})$ & $k_{e 0 \_ \text {estimation }} 0.187$ & $k_{e 0 \_ \text {tpeak }}: 1.2152$ \\
Target effect-siteconcentration of propofol $(\mu \mathrm{g} / \mathrm{ml})$ & $1.0-2.6$ & $2.0-3.0$ \\
$k_{e 0}$ for simulation based on infusion history & Time-to-peak effect: $1.68 \mathrm{~min}$ & $k_{e 0 \_ \text {estimation: }}: 0.26 / \mathrm{min}$ \\
\hline
\end{tabular}

LCT: long-chain triglyceride, $k_{e 0_{e} \text { estimation }}$ estimated from sequential pharmacokinetic/pharmacodynamic modeling of a single population, $k_{e 0 \text { _tpeak }}$ : calculated using the observed time-to-peak effects. 
median (range: 25-75\%) for nonnormally distributed continuous variables, and as counts and percentages for categorical variables. The relationship between age, time-to-peak effects, and BIS was analyzed using linear regression. All $\mathrm{P}$ values $<0.05$ were considered statistically significant.

\section{Results}

All patients enrolled in the study of obtaining $k_{e 0}$ of microemulsion propofol calculated by $t_{\text {peak }}$ method were included in order to analyze the time to peak effect. Time course of BIS values after an intravenous bolus injection of microemulsion propofol $1 \mathrm{mg} / \mathrm{kg}$ are shown in Fig. 1. Median (25-75\%) value of the time to maximally reduced BIS from baseline value was 1.68 (1.24-2.45) $\mathrm{min}$. Also, relationship between age and time to peak effect in BIS are presented in Fig. 2, where, with increasing age, time to peak effect $\left(\mathrm{t}_{\text {peak }}\right)$ increased.

A total of 1,027 BIS values (average value: $63.3 \pm 16.9$, sampling duration: $17.1 \pm 3.5 \mathrm{~min}$ ) from 20 patients receiving $\mathrm{mi}-$ croemulsion propofol and 1,878 BIS values (average value: 59.3 \pm 17.7 , sampling duration: $24.0 \pm 7.2 \mathrm{~min}$ ) from 20 patients receiving LCT propofol were used to determine predictive performance in BIS prediction. Performance error (PE) and absolute performance error (APE) in BIS values for microemulsion propofol and LCT propofol according to the different $k_{e 0}$ s were depicted in Fig. 3. PE of $k_{\text {en_estimation }}$ was significantly lower than that of $k_{e 0 \_ \text {tpeak }}$ in microemulsion propofol ( $\mathrm{P}=0.013$, Mann-Whitney rank sum test). APE of $k_{e 0 \text { estimation }}$ showed significant differences in both formulations, compared with $k_{e 0 \_ \text {tpeak }}(\mathrm{P}<0.001$, MannWhitney rank sum test). Bias (median performance error) and inaccuracy (absolute performance error) in BIS prediction values for microemulsion and LCT propofol in same pharmacoki-

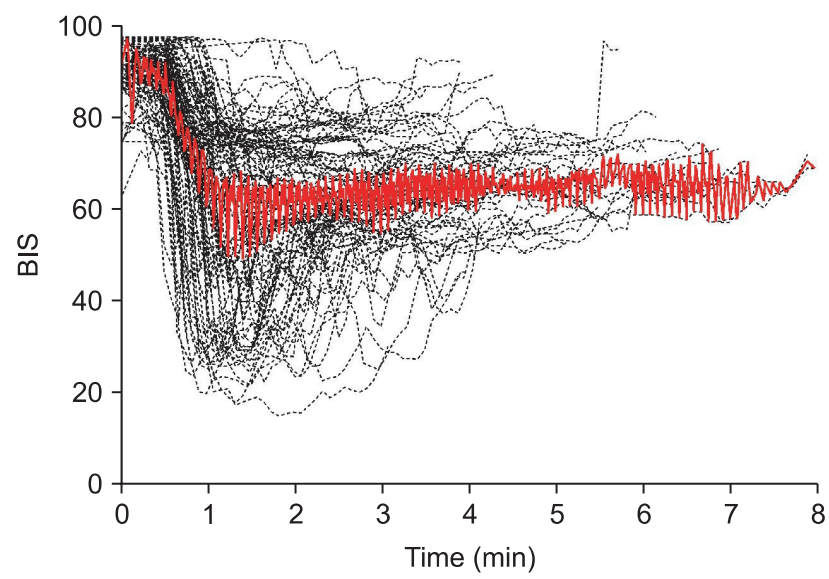

Fig. 1. Raw recordings of the bispectral index (BIS) in 100 patients who received an intravenous injection of $1 \mathrm{mg} / \mathrm{kg}$ microemulsion propofol. The thick lines and black dashed lines indicate the mean and observed BIS values of individual patients, respectively. netic model with different $k_{e 0}$ s are demonstrated in Table 4. BIS values predicted by $k_{\text {en_estimation }}$ method showed higher accuracy than those predicted by $k_{e 0 \_ \text {tpeak }}$ method.

\section{Discussion}

The median $t_{\text {peak }}$ of microemulsion propofol was $1.68 \mathrm{~min}$. The $k_{e 0}$ derived from sequential pharmacokinetic and pharmacodynamic modeling showed higher accuracy in BIS prediction than that of $k_{e 0}$ calculated by the time to peak effect in both propofol formulations.

The $t_{\text {peak }}$ method should be used only for linking separate pharmacokinetic and pharmacodynamic studies for the same drug, as employed in an earlier study [1], where $t_{\text {peak }}$ increased with age and this finding was consistent with our results. In other study [10], $\mathrm{t}_{\text {peak }}$ was not affected by age, which may be explained by the fact that distribution of age was relatively narrow ( $49 \pm 16 \mathrm{yr}$ ). Age of patients enrolled in present study for obtaining $k_{e 0}$ of microemulsion propofol calculated by $t_{\text {peak }}$ method was 24-86 yr, which was similar to the previous study (25-81 yr) [1]. Overall, elderly patients showed prolonged onset of action on administration of drug [11,12]. Age was a significant covariate for $k_{e 0}$ in population pharmacodynamic modeling of remifentanil, where $\mathrm{t}_{2 / 1} k_{e 0}$ increase with increasing age $[7,13]$. These facts may be explain the relationship between age and time to peak effect, even though effect of age on $k_{e 0}$ of propofol using population pharmacodynamic analysis has not yet been elucidated.

In Noh model, lean body mass, weight and sex were significant covariates for $V_{2}, C L$ and $Q_{1}$, respectively, resulting in different hybrid constants according to the individual patients. Hence, $k_{e 0} \mathrm{~s}$ of microemulsion propofol calculated by $\mathrm{t}_{\text {peak }}$ method have different values when height, weight and sex are not identical

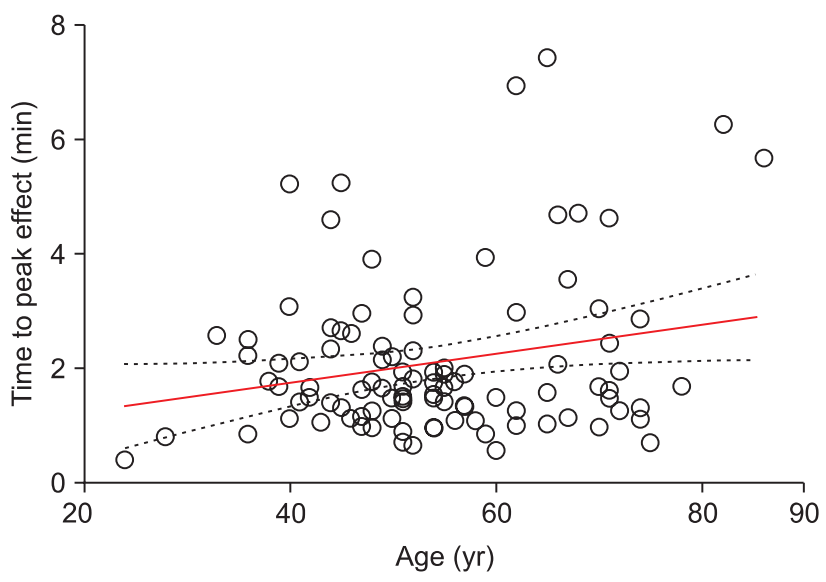

Fig. 2. Relationship between age and time-to-peak effects on the bispectral index (BIS). The solid lines and black dashed lines indicate the regression line and $95 \%$ confidence intervals, respectively. Time-to-peak effect $=0.03 \times$ age $($ years $)+0.74$ (simple linear regression; $\mathrm{P}=0.03$ ) . 

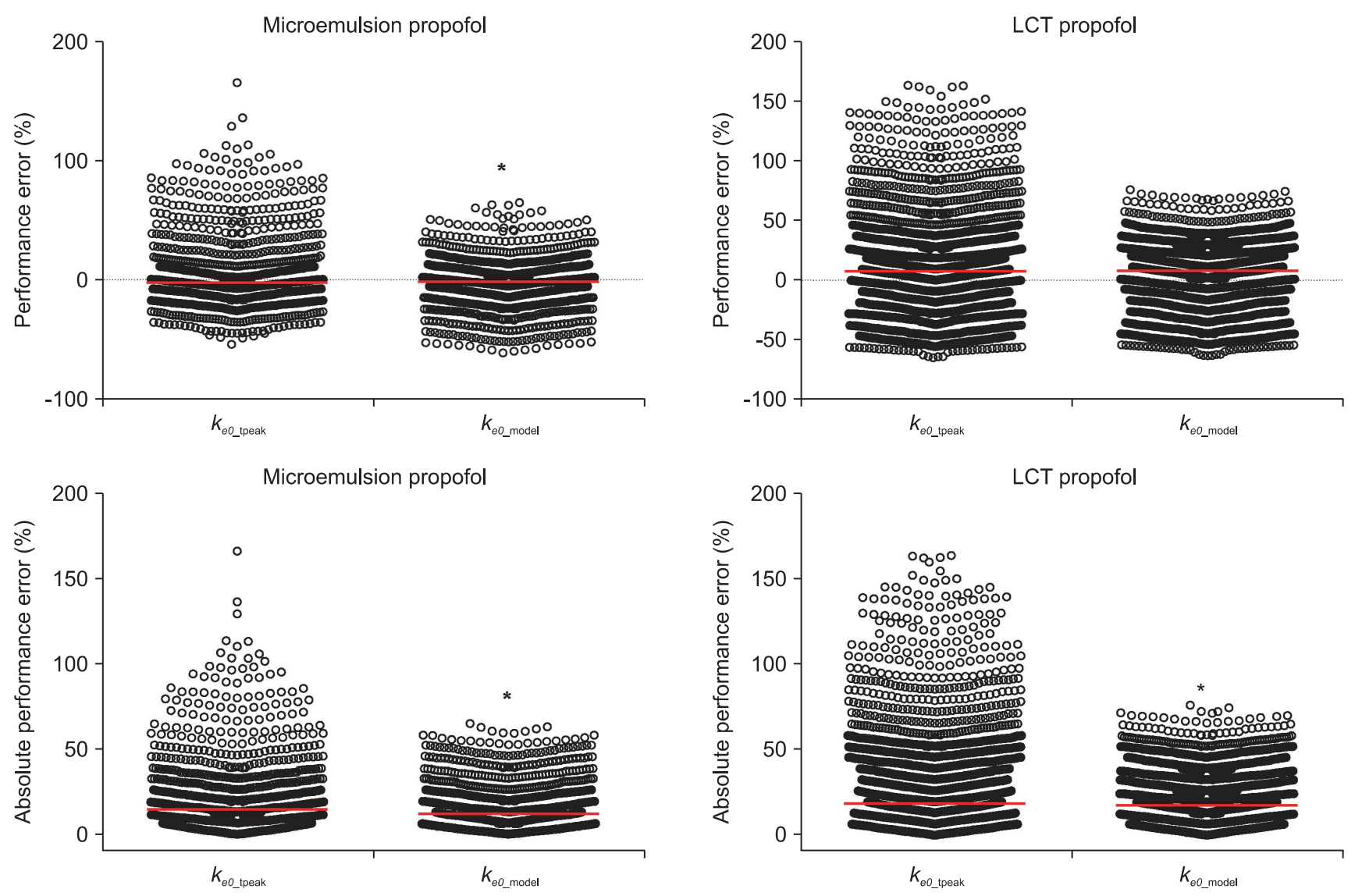

Fig. 3. Performance error and absolute performance error of the bispectral index (BIS) values for microemulsion propofol and long-chain (LCT) triglyceride propofol according to $2 k_{e 0} \mathrm{~s}$ values. $k_{e 0 \_ \text {model }}$ estimated from sequential pharmacokinetic/pharmacodynamic modeling of a single population, $k_{e 0_{-} \text {tpeak }}$ : calculated using the observed time-to-peak effects. The values of the $k_{e 0 \_ \text {model }}$ and $k_{e 0 \_ \text {tpeak }}$ models were $0.187 / \mathrm{min}$ and 1.68 min (time-to-peak effects) for microemulsion propofol and $0.26 / \mathrm{min}$ and $1.2152 / \mathrm{min}$ for LCT propofol, respectively. $* \mathrm{P}<0.05$ vs $k_{e 0 \_ \text {tpeak- }}$. Performance error (PE) was calculated as follows:

$$
\mathrm{PE}_{\mathrm{ij}}=\frac{\text { measured BIS }_{\mathrm{ij}}-\text { predicted BIS } \mathrm{ij}_{\mathrm{ij}}}{\text { predicted BIS }}
$$

Predicted BIS was calculated using the following equations. For microemulsion propofol,

$$
\text { Predicted BIS }=87.8+(22.2-87.8) \frac{\text { effect-site concentration }}{3.05} \frac{1.58^{3.05}+\text { effect-site concentration }}{3.05} \text {. }
$$

For LCT propofol,

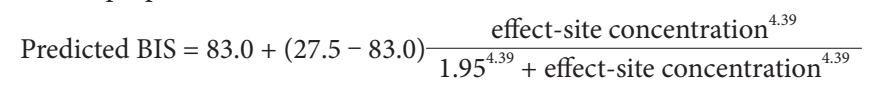

The pharmacodynamic parameters for microemulsion and LCT propofol that were used to calculate the predicted BIS values were obtained in previous studies. Empty circles $(\mathrm{O})$ and red horizontal lines indicate the performance error of each BIS measurement and the median values,

\begin{tabular}{|c|c|c|c|c|}
\hline & \multicolumn{2}{|c|}{ Microemulsion propofol $(\mathrm{n}=20)$} & \multicolumn{2}{|c|}{ LCT propofol $(\mathrm{n}=20)$} \\
\hline & $k_{e 0 \_ \text {model }}$ & $k_{e 0 \_ \text {tpeak }}$ & $k_{e 0 \_ \text {model }}$ & $k_{e 0_{-} \text {tpeak }}$ \\
\hline Bias (MDPE), \% (95\% CI) & $-1.83(-24.81$ to 18.95$)$ & $-2.42(-26.10$ to 36.18$)$ & $8.01(-20.55$ to 30.06$)$ & $7.37(-27.03$ to 49.10$)$ \\
\hline Inaccuracy (MDAPE), \% (95\% CI) & $11.87(2.16$ to 31.13$)$ & $14.38(-0.61$ to 44.60$)$ & $17.31(5.54$ to 35.98$)$ & $18.28(-0.10$ to 56.04$)$ \\
\hline
\end{tabular}
respectively.

Table 4. Comparison of the Biases and Inaccuracies of Bispectral Index Prediction

LCT: long chain triglyceride, MDPE: median performance error, MDAPE: median absolute performance error, $k_{e 0 \text { model }}$ : estimation from sequential pharmacokinetic-pharmacodynamic modeling in a single population, $k_{e 0 \_ \text {tpeak }}$ : calculation using the observed time to peak effect. The value of $k_{e 0 \_ \text {model }}$ and $k_{e 0} t_{\text {peak }}$ for microemulsion propofol and LCT propofol were $0.19 / \mathrm{min}$ and $1.68 \mathrm{~min}$ (time to peak effect) for microemulsion propofol and $0.26 / \mathrm{min}$ and $1.21 / \mathrm{min}$ for LCT propofol, respectively. 
each other. On the other hand, in modified Marsh model, weight was a significant covariate for all pharmacokinetic parameters in volume and clearance domain, resulting in same hybrid constant due to reducing a fraction with weight. The $k_{e 0}$ of LCT propofol calculated by $t_{\text {peak }}$ method is identical $(1.2152 / \mathrm{min})$ in modified Marsh model, irrespective of patients' demographics.

In general, predictive performance of a TCI system was clinically acceptable when inaccuracy is less than 20-30\% [9]. Pharmacodynamic predictions as described by BIS exhibited smaller bias and inaccuracy in present study. Theoretically, because effect-site concentration per se should be statically related to effect, target effect-site controlled infusion using Noh or modified Marsh models can be available to predict BIS approximately, irrespective of method for obtaining $k_{e 0}$.

Biases and inaccuracies of a TCI system may be smallest when it incorporates pharmacokinetic parameters and $k_{e 0}$ obtained from a single population. Potential causes of performance errors in the predictive performance of a TCI system may include model misspecifications and mechanical errors of the infusion pump, such as trumpet curves [14]. A trumpet curve could be more likely to develop on long time of infusion. In this study, effect of trumpet curve can be excluded from interpretation of result, because predictive performance of BIS prediction was analyzed in induction and recovery periods. Model misspecification of pharmacodynamic model shows a tendency of being smaller in the sequential pharmacokinetic and pharmacodynamic modeling, compared with simultaneous pharmacokinetic and pharmacodynamic modeling. Model misspecification of pharmacokinetic model can convert to that of pharmacodynamic model in simultaneous pharmacokinetic and pharmacodynamic modeling. Pharmacokinetics of microemulsion propofol were well described by mammillary three compartment model and the results of typical pharmacokinetic parameter were used to characterize the relationship concentration and effect using inhibitory sigmoid Emax model.

In summary, the median time to maximally decreased BIS from baseline value after an intravenous bolus of microemulsion propofol $1 \mathrm{mg} / \mathrm{kg}$ was $1.68 \mathrm{~min}$. BIS values predicted by $k_{\text {e0_estimation }}$ method showed higher accuracy than those predicted by $k_{e 0 \_ \text {tpeak }}$ method.

\section{References}

1. Schnider TW, Minto CF, Gambus PL, Andresen C, Goodale DB, Shafer SL, et al. The influence of method of administration and covariates on the pharmacokinetics of propofol in adult volunteers. Anesthesiology 1998; 88: 1170-82.

2. Minto CF, Schnider TW, Gregg KM, Henthorn TK, Shafer SL. Using the time of maximum effect site concentration to combine pharmacokinetics and pharmacodynamics. Anesthesiology 2003; 99: 324-33.

3. Marsh B, White M, Morton N, Kenny GN. Pharmacokinetic model driven infusion of propofol in children. Br J Anaesth 1991; 67: 41-8.

4. Struys MM, De Smet T, Depoorter B, Versichelen LF, Mortier EP, Dumortier FJ, et al. Comparison of plasma compartment versus two methods for effect compartment--controlled target-controlled infusion for propofol. Anesthesiology 2000; 92: 399-406.

5. Jung JA, Choi BM, Cho SH, Choe SM, Ghim JL, Lee HM, et al. Effectiveness, safety, and pharmacokinetic and pharmacodynamic characteristics of microemulsion propofol in patients undergoing elective surgery under total intravenous anaesthesia. Br J Anaesth 2010; 104: 563-76.

6. Kim YH. Nonlinear pharmacokinetics and clinical implications of blood-brain equilibration half-time of propofol (Master's dissertation). Seoul: Ulsan University of College of Medicine, 2011.

7. Minto CF, Schnider TW, Egan TD, Youngs E, Lemmens HJ, Gambus PL, et al. Influence of age and gender on the pharmacokinetics and pharmacodynamics of remifentanil. I. Model development. Anesthesiology 1997; 86: 10-23.

8. Billard V, Gambus PL, Chamoun N, Stanski DR, Shafer SL. A comparison of spectral edge, delta power, and bispectral index as EEG measures of alfentanil, propofol, and midazolam drug effect. Clin Pharmacol Ther 1997; 61: 45-58.

9. Varvel JR, Donoho DL, Shafer SL. Measuring the predictive performance of computer-controlled infusion pumps. J Pharmacokinet Biopharm 1992; 20: 63-94.

10. Zhang MZ, Yu Q, Huang YL, Wang SJ, Wang XR. A comparison between bispectral index analysis and auditory-evoked potentials for monitoring the time to peak effect to calculate the plasma effect site equilibration rate constant of propofol. Eur J Anaesthesiol 2007; 24: 876-81.

11. Vuyk J. Pharmacodynamics in the elderly. Best Pract Res Clin Anaesthesiol 2003; 17: 207-18.

12. Ornstein E, Lien CA, Matteo RS, Ostapkovich ND, Diaz J, Wolf KB. Pharmacodynamics and pharmacokinetics of cisatracurium in geriatric surgical patients. Anesthesiology 1996; 84: 520-5.

13. Noh GJ, Kim KM, Jeong YB, Jeong SW, Yoon HS, Jeong SM, et al. Electroencephalographic approximate entropy changes in healthy volunteers during remifentanil infusion. Anesthesiology 2006; 104: 921-32.

14. Schraag S, Flaschar J. Delivery performance of commercial target-controlled infusion devices with Diprifusor module. Eur J Anaesthesiol 2002; 19: 357-60. 